\title{
Evaluation of clinical symptom, laboratory finding and prognosis in thrombotic thrombocytopenic purpura patient from 2010 t0 2017. A single-center retrospective study
}

\author{
Mohammad- Reza Rostami \\ Tehran University of Medical Sciences \\ Amirmasoud Kazemzadeh Houjaghan \\ Tehran University of Medical Sciences \\ Sahar Tavakoli shiraji ( $\nabla$ stavakoli@sina.tums.ac.ir ) \\ Tehran University of Medical Sciences \\ Hosein Kamranzadeh Fumani \\ Tehran University of Medical Sciences
}

Research

Keywords: clinical symptom, Laboratory finding, prognosis

Posted Date: April 2nd, 2020

DOl: https://doi.org/10.21203/rs.3.rs-19605/v1

License: (c) (1) This work is licensed under a Creative Commons Attribution 4.0 International License.

Read Full License 


\section{Abstract}

Background and purpose: thrombotic thrombocytopenic purpura (TTP) is associated with microangiopathic hemolytic anemia, thrombocytopenia, and micro vascular thrombus plus fever, fluctuating neurologic abnormalities and renal damage. The purpose of this study was to investigate clinical symptom, laboratory finding and prognosis in TTP patient from 2010 to 2017 in Shariati hospital. In the present study we assessed clinical presentation and laboratory finding to predict mortality in patients with TTP.

Methods: the study was a retrospective cohort study in patient with TTP in Shariati hospital from 2010 to 2017. 114 subjects with TTP after rule out of other microangiopathic hemolytic anemia were included in this study.

Results: 114 case of TTP ( 80 female and 34 male) were identified. Mean ages of participated in the study were 39 years. Hematologic and neurologic symptoms were most common manifestations. Laboratory result at the time of diagnosis revealed mean thrombocytopenia (Plt: 29100), anemia ( $\mathrm{Hb}: 8 / 1)$, elevated LDH (15100). all patient were treated with PEX, using a cut-off value for the platelet count of $150000 / \mathrm{ml}$. $75 \%$ of patient responded . In this study mean of ages, Neurologic manifestation, category of immunologic and reticulocyte count predicted mortality.

Conclusion: finally, it can be concluded that mortality rate similar to other study but: in difference clinical symptom and laboratory finding may be predict mortality and occurrence of relapse.

\section{Introduction:}

In recent years, the close relationship between an old disease called thrombotic thrombocytopenic purpura (TTP) and a deficiency in a new protein called ADAMTS13 has been highly regarded ( 2 and 1 ). The disease is a rare hematological disease with an annual prevalence of about 10 cases per million and an annual incidence of about one new case per million (3-11). The first acute episode of the disease occurs predominantly during adulthood (which accounts for $90 \%$ of patients), but some children and adolescents also experience the initial episode $(13,12)$. The disease is mainly caused by an autoimmune mechanism but non-autoimmune but familial forms of the disease have also been described (called Upshaw-Schulman syndrome). TTP is twice more common in women than in men, and even recurrence is more common in women. The disease continues to be a fatal disease with mortality of over 10 to 20 percent despite recent advances in treatment protocols (14).

The definition of TTP has changed a lot over time. Early on, the acute episode of TTP was defined on the basis of clinical evidence (mainly visceral ischemic symptoms mainly focused on the brain) as well as standard biological parameters (including microangiopathic hemolytic anemia and severe thrombocytopenia) in the absence of other causes. In this definition, the role of ADAMTS13 deficiency has also been added in recent years, being the only diagnostic marker specific for TTP (14). Severe defect in ADAMTS13 causes the accumulation of von Willebrand factor multimers in the blood resulting in the 
formation of platelet-rich microtombuses, especially in the small and distal arterioles (14). In most cases, the mechanism of severe ADAMTS13 defect is explained by the release of autoantibodies against ADAMTS13 mainly during the acute phase of the disease. These anti-ADAMTS13 lgGs antibodies usually inhibit the proteolytic activity of ADAMTS13 against von Willebrand factor, and therefore significant amounts of the circulating immune complex associated with ADAMTS13 or ADAMTS13-ICs are visible in TTP-acquired forms (25).

In clinical presentation, fivefold incidence of fever, thrombocytopenia, microangiopathic hemolytic anemia, neurologic symptoms, and renal insufficiency have been shown to define and diagnose TTP, but many reports have reported incidence of these five in less than 10\% of TTP patients ( 44 and 10). However, persistent signs of the disease, including thrombocytopenia, microangiopathic hemolytic anemia (based on the observation of peripheral blood smears) have maintained their role in the diagnosis. Symptoms associated with ischemia or organ infarction are predominantly brain related, with manifestations of headache, convulsions, stroke and even coma. Cardiac ischemia (in 25\% of patients) as well as mesenteric ischemia (in 35\%) is seen in patients (45). Symptoms of renal insufficiency in the form of hematuria, proteinuria, although acute renal failure is usually rare, and so acute acute renal failure is sometimes seen in severe cases of TTP up to $10 \%$ to $27 \%$ (46). Many patients also have symptoms completely unrelated to the pathophysiology of TTP, including bacterial infections or autoimmune diseases (such as SLE), medication use (cyclosporine, quinine, clopidogrel and ticlopidine), HIV infection, puncture, HIV Cancer types noted. In this regard, it is important to pay attention to the specificity or deficiency of ADAMTS13 in TTP to distinguish it from other diseases.

Concerning the prognosis of TTP, the survival rate of patients with TTP has been estimated at $80 \%$ to $90 \%$ since the first episode of the disease (64). High age, high levels of lactate dehydrogenase (above 10 times the normal level), organ damage associated with the disease, and elevated cardiac troponin levels have all been associated with poor prognosis and failure to respond to treatment (66 and 65). In spite of clinical examinations and normal laboratory tests in some patients, non-recovery following treatment in patients has been described $(68,67)$. Neurologic deficits, arterial hypertension, and major depression are commonly reported in these patients even after complete treatment. In addition, patients with TTP also struggle with certain connective tissue diseases such as SLE and Sjogren's syndrome. In $40 \%$ of patients, patients experience one or more recurrences in the long term (69). It should be noted that evaluation of ADAMTS13 activity was the only important factor in predicting and tracking recurrence even in the long run. Regarding treatment response criteria, complete response to treatment approaches is generally considered in the form of platelet counts above 150 for two consecutive days with normal LDH levels and complete resolution of clinical symptoms. Treatment response is expected at least 30 days after TPE discontinuation. In this regard, refractory disease is defined as exacerbation or recurrence within 30 days or more after treatment protocol.

In a study by Chaturuedi et al., between 2000 and 2012, a cohort study was performed to monitor the outcome of patients with TTP at the Cleveland Clinic. No fever, neurological problem, or renal impairment. Other cases of microangiopathic hemolytic anemia (from DIC, Sebis, Eclamysi, etc.) were excluded. 
Patients diagnosed with TTP according to underlying cause in six autoimmune, transplanted, Malignancies were associated with delivery, drug, or idiopathic. Clinical outcomes of treatment in response to It was defined as $<15,000<$ plt and normal LDH and recurrence was lower than platelets below 15,000 and increased LDH following a defined initial response was assessed and analyzed using the REDCAP tool. All variables were analyzed for outcome evaluation. Classified variables were analyzed with Fisher exact test, Pearson chi square and continuous data with Wilcoxon signed and t-test. $P$ value $<0.05$ was considered significant in the analyzes. Increased age, especially over 60 years $(P=0.002 \mathrm{Cl}$ 95\% 2015-23 RR: 0.8,) Severe neurological symptoms (P <0.001 , Cl95\% -14-80 RR: 18/37 (and high LDH after two-dose plasma exchange predicted mortality (98).

In a 2009 study by N.Frawley et al., A retrospective study entitled thrombocytopenic thrombotic purpura has a high association with recurrence after plasma replacement in a single-center experience, which retrospectively reviewed 400 patients between 2005 and 1992 at the hospital. The patients in this study were defined with microangiopathic hemolytic anemia and thrombocytopenia with or without renal involvement, neurology, and fever. Patients treated with FFP were treated once daily with plasma FFP. Obtained from patients and pathological evidence the goal of not having complete response and recurrence after complete treatment of partial PR response and refractory disease or death during the TTP period was defined. CR was confirmed as platelet and LDH normalization, hemoglobin elevation, and microangiopathy and neurological symptoms except stroke. Sustained CR was defined as non-recurrent within 1 month, and refractory disease was defined as one of the following. Platelets were less than 75,000 either hemolysis or death before one week of treatment, and PR was defined as biochemical and hematologic improvement with platelets above 75,000,000,000 within 2 weeks of treatment. The results of the study showed that 31 patients achieved CR with an average of 11 times plasma and steroid replacement.

\section{Methodology:}

Type of Study: This was a retrospective cohort study.

Study population: Patients with TTP referred to Dr. Shariati Hospital during 2010-2010

In the methodology, patients are divided into the following idiopathic and immunologic subgroups, including Lupus, Wegener, and rheumatoid arthritis, in relation to pregnancy from pregnancy to one week after pregnancy and in relation to concussion and medication. Clinical manifestations include hematologic manifestations. Petechiae and purpura, gastrointestinal bleeding, hemoptysis, and neurologic manifestations including seizures, intracerebral hemorrhage, and laboratory manifestations including platelet loss during hospitalization without clinical symptoms, fever and jaundice, and creatinine elevation of more than $3 \%$ of basal levels.

Methods: This study was a retrospective cohort study in which the researcher referred to hospital records, telephone calls and face-to-face interviews to evaluate clinical and laboratory symptoms and prognosis of patients with TTP admitted to Dr. Shariati Hospital during 2010. By 1396 he had paid. The researcher 
referred to the Plasma Unit of Shariati Hospital after receiving the authorization and introducing a letter from the Vice Chancellor for Research, Shariati University of Medical Education, Research and Therapeutic Center. Data was collected on patients with TTP who were treated at the center. Data collection tools were referral to hospital and clinic records, telephone contact with patients, and face-toface interviews with patients and their first-degree relatives. Through the above, the researcher gathers information on demographic characteristics, initial tests, clinical symptoms, examination findings, treatments performed, known underlying or later diagnosed diseases, laboratory follow-up, number and The type of treatment performed, the disease resistance and relapse, and ultimately the long-term survival of the patients.

2 Statistical Analysis Method: The results were expressed as mean and standard deviation (mean \pm SD) for the quantitative variables and as percentages for the qualitative variables. Data were analyzed using t-test or ANOVA and chi-square test was used to compare qualitative variables. Multivariate logistic regression model was used to determine factors related to patient survival or relapse. Also, the long-term survival of patients was determined using the Kaplan-Meier curve. Significance level was considered less than 0.05 . SPSS software version 23 was used for statistical analysis.

Ethical considerations: Patients' private information is kept strictly confidential. There was no disruption in their diagnostic and therapeutic course and no additional cost was imposed on the patient. Any use of patient information was obtained with the permission of the researcher and confirmed by the research assistant of Tehran University of Medical Sciences.

\section{Discussion:}

The epidemiological aspects of TTP have been studied in different societies, but the epidemiological features of these patients in Iranian society have not been thoroughly investigated so far. Evaluation of various epidemiological, clinical, diagnostic and also long-term therapeutic implications of patients is essential for macro-planning because the disease is associated with high mortality and disability rates and strongly underpins psychological and quality of life of patients. Overwhelms. What we did in the present study was primarily to evaluate the epidemiological aspects, clinical manifestations, laboratory findings as well as short and long term outcomes of patients with TTP in Iranian society and then to evaluate the relevant and influencing factors. We evaluated the long-term survival of patients and the long-term relapse of the disease in Iranian society. Epidemiologically, we have shown that the sex distribution of TTP in women is far greater than in men (with a ratio of approximately 3 to 1 ) and the mean age of patients is about 39.3 years, which is quite consistent with reports from other societies. . In Swart et al.'s study, $78.1 \%$ of patients were female (91). In the study of Alwan et al., $68 \%$ of patients were female and the median age of patients was 46 years (93). In Iqbal et al.'s study, 18 (75\%) patients were female and the mean age was 33.5 years (94). In the study of El-Husseiny et al., The female frequency was $56.6 \%$ and the median age was 42 years (97). Therefore, in terms of sex distribution as well as middle age, our country was similar to other countries. In terms of clinical presentation, the most common manifestations in our patients included hematologic signs and symptoms, neurological 
disorders, as well as routine laboratory manifestations that were consistent with other studies. In the study of lqbal et al., In general, the most common manifestations included neurological lesions, renal impairment, and TTP diagnostic laboratory triad (94). In the study of Wang et al., Among 14 patients, 14 patients had neurologic symptoms, hemolytic anemia, and decreased platelet count. Eight patients also had fever and eight patients had renal impairment (95). In the study of Sun et al., Common symptoms were thrombocytopenia in $100 \%$, hemolytic anemia in $92.1 \%$, neurologic symptoms in $88.2 \%$, fever in $72.5 \%$ and renal impairment in $70.5 \%$ (96). In the study of Chaturuedi et al., Diagnostic symptoms included microangiopathic hemoptic anemia, thrombocytopenia with or without fever, neurological problems, or renal impairment (98). Therefore, in all TTP patients, the occurrence of microangiopathic hemolytic anemia associated with thrombocytopenia, neurological disorders as well as renal disorders will be prominent findings.

Regarding the therapeutic approaches planned for patients, almost all TTP patients receive either plasma or PEX replacement therapy, and supplemental treatment with steroids or rituximab is also considered to reduce the effects of PEX. In our patients, all were treated with PEX and also $10.5 \%$ of patients received rituximab. This treatment approach had a favorable outcome as far as response rate to treatment and recovery rate, complete recovery in $63.2 \%$, partial remission in $12.3 \%$ and no remission in $24.6 \%$ were reported. On this basis, the overall rate of recovery after treatment was estimated to be $75.5 \%$. Also, the prevalence of recurrence was estimated to be $21.1 \%$. Overall, the prevalence of mortality was estimated to be $26.3 \%$, which is consistent with studies in other communities. In Swart et al.'s study, primary disease treatment included PEX in $87.8 \%$, plasma infusion in $78.1 \%$, antiviral treatment in $78.3 \%$, steroids in $61 \%$, admission to intensive care unit in $41.5 \%$, dialysis Kidney was $12.2 \%$ and other immunosuppressive drugs was $4.9 \%$. The recurrence rate after treatment was $9.8 \%$ and the mortality rate was $29.3 \%$ (91), which was very close to the results of our study. In the study of Alwen et al., Mortality rate was $10.3 \%$, which was less reported than in our study (93). In the study of Iqbal et al., All patients received PEX, 95.8\% had steroids, and $54 \%$ had received ritoximab. Complete recovery was reported in $87.5 \%$. At the 22 -month follow-up, $83.3 \%$ were life-threatening, and the mortality rate was estimated to be $16.7 \%$, which was slightly lower than our study (94) because in our center Ritoximab used lower due to lack of financial support. In Wang et al.'s study, after treatment with PEX, steroids, and rituximab, $85.7 \%$ of patients were successfully treated and $14.3 \%$ died, but what was of particular interest in their study was the high incidence of two-year relapse in patients It was $66.7 \%$ (95). In the study of Sun et al., PTE treatment was associated with response in $72.3 \%$. Among 36 patients, $22.2 \%$ had recurrence and $29.4 \%$ had died (96), which was very close to our results. Therefore, what our study showed was a significant improvement in patients over the long term and a decrease in the recurrence rate.

Finally, what we found in our study was that factors predicting long-term survival of patients included older age and the appearance of neurological manifestations. Also, in predicting long-term recurrence, lower age, immunological origin for the disease, and decreased platelet count were predictive factors for long-term recurrence in patients. Various studies have been reported to predict mortality and recurrence. In the study of Staley et al., Failure to normalize platelet counts increased LDH levels, decreased protein or albumin levels, and increased serum troponin levels were predictors of mortality (92). In the study of 
Alwan et al., Mortality rate was associated with increased levels of anti-ADAMTS13 antibody and decreased ADAMTS13 antigen (93). In El-Husseiny et al.'s study, predictors of recurrence of disease included decreased platelet count and increased LDH levels (97). In Chaturuedi et al.'s study, increasing age, especially over age 60, severe neurologic symptoms, and elevated LDH after two-second plasma exchange were predictive of mortality (98), which is consistent with our study.

In the study of N.Frawley et al., Neurological symptoms were the only predictor of treatment failure (99). In this study, however, we focused on epidemiological and clinical factors rather than laboratory and diagnostic findings. Therefore, in summing up the factors associated with first-line treatment failure and therefore predicting long-term mortality and recurrence, in addition to age factors, neurological complications, underlying immunological abnormalities, significant reduction in platelet count as well as reticulocytes as well as reduction ADAMTS13 activity and elevated serum LDH levels should be considered at a glance. Therefore, a set of new scoring systems for predicting outcome or outcome of patients can be used in combination of these factors.

\section{Results And Findings:}

Basic characteristics of patients with TTP:

In this study, a total of 114 patients with TTP were studied. In terms of sex distribution, 80 cases (70.2\%) were female and 34 cases $(29.8 \%)$ were male. The mean age was $39.3+14.9$ years ranging from 21 to 84 years. In total, 83 patients $(72.8 \%)$ were outpatient and 31 cases $(27.2 \%)$. In terms of disease classification, $47(41.2 \%)$ were idiopathic, $21(18.4 \%)$ pregnancy-type, $31(27.2 \%)$ immunologic, 7 The case $(6.1 \%)$ was followed by transplantation, in 6 cases $(5.3 \%)$ it was cancerous and in 2 cases (1.8\%) it was drug induced. In terms of disease manifestations, hematologic manifestations in 64 cases (56.1\%), neurologic manifestations in 24 cases $(21.1 \%)$, routine laboratory manifestations in 22 cases $(19.3 \%)$, jaundice in 2 cases (1.8\%) \%), Fever in 1 case $(0.9 \%)$ and increased creatinine level in 1 case $(0.9 \%)$. In terms of laboratory presentation, mean hemoglobin was $9.58 \pm 8.65 \mathrm{~g} / \mathrm{dl}$, mean creatinine was $1.77 \pm$ $1.55 \mathrm{mg} / \mathrm{dl}$, mean LDH level was $189.89 \pm 1293.77 \mathrm{mg} / \mathrm{dl}$. , Mean Schistosite count was 5.03 /1 1.27, mean platelet count was $36491.23+25836.53 \mathrm{~mm} 3$, mean AST level was $60.41+56.24 \mathrm{mg} / \mathrm{dl}$, mean ALT level was $94.64+-2.76 \mathrm{mg} / \mathrm{dl}$, mean total bilirubin level was $2.76+2.29 \mathrm{mg} / \mathrm{dl}$ and mean direct bilirubin level was $2.76 \pm 2.28 \mathrm{mg} / \mathrm{dl}$. Mean reticulocyte count was $5.43+3.79$. In the treatment protocol, all patients underwent PEX over an average of 16.61 rounds. Also, 12 (10.5\%) patients received rituximab. Three patients received vincristine in total.

3.2.2 Outcome information for patients with TTP: 
In terms of response to treatment and recovery, complete remission was reported in 72 cases (63.2\%), partial remission in 14 cases (12.3\%) and no remission in 28 cases (24.6\%). On this basis, the total recovery rate after treatment was estimated to be 86 (75.5\%). The incidence of recurrence was estimated to be 24 (21.1\%) in total. Overall, the prevalence of mortality was 30 (26.3\%). Mean CR was $31.17 \pm 31.34$ months, mean platelet count normalization time was $15.24 \pm 18.42$ days and mean recurrence time was $10.56+28.57$ months.

\section{Calculating the survival of patients with TTP:}

Based on Kaplan-Meier curve analysis of survival, 30-day survival was $82.5 \%, 76.3 \%$ one-year survival, $74.6 \%$ two-year survival, and $73.7 \%$ three-year survival. Five years was $2.71 \%$. In terms of recurrence-free survival, 6-month recurrence-free survival was $93.8 \%$, one-year recurrence-free survival equal to $89.3 \%$, two-year recurrence-free survival equal to $89.3 \%$, and three-year recurrence-free survival equal to $6.85 .5 \%$ and five-year recurrence-free survival were $2.83 \%$.

Determination of Predictors of Mortality in Patients:

In both groups, the frequency of death was $60(71.4 \%)$ and $20(66.7 \%)$, respectively (P value $=0.625)$. The mean age of the two groups was $34.89+10.14$ years and $51.67+19.17$ years, respectively, which was significantly higher in the deceased group ( $P$ value less than 0.001$)$. According to the distribution of disease category in the two groups, the prevalence of idiopathic type was 39 cases (46.4\%) and 8 cases $(26.7 \%)$ respectively, the frequency of pregnancy type was 20 cases (23.8\%). ) And 1 case (3.3\%), frequency of immunological type was 20 cases (23.8\%) and 11 cases (36.7\%), frequency of transplant type was 3 cases (3.6\%) and 4 cases ( 3 cases). (13\%), malignant type was $0(0 \%)$ and $6(20 \%)$ and the frequency of drug type was $2(2.4 \%)$ and $0(0 \%)$, respectively. There was a significant difference between the two groups in that the frequency of malignancy was significantly higher in the deceased group $(P$ value less than 0.001 ). In terms of clinical manifestations, the incidence of neurologic manifestations was $12(14.3 \%)$ and 10 (40.0\%), respectively (55.5\%) in both groups. And 9 cases (30\%), jaundice occurrence 0 (0\%) and 2 cases (6.7\%), fever occurrence $0(0 \%)$ and 1 case (3.3) \%), Laboratory presentation was 16 (19\%) and 6 (20\%) and creatinine increased 1 (1.2\%) and 0 (0\%), respectively, indicating higher prevalence. Neurologic manifestations were higher in patients $(P$ value $<0.001)$. In the group of deceased patients, mean hemoglobin was $8.13+1.79$ and 10/10 \pm 9.98 ( $P$ value $=0.228)$, mean creatinine was $2.13+1.34$ and 0.60 , respectively. 1.611 (P value $=0.146)$, mean LDH was 2296/60+ 1513.03 and $2112.78+1213.83(P$ value $=0.601)$, mean platelet count was $34100 / 00+29520 / 71(P$ value $=0.557)$, AST mean $50.5 \pm 31.8$ and $63.8+62.5(P$ value 0.27$)$, ALT mean $94.64 \pm 57.92(P$ value 0.372 ), mean total bilirubin $2.76 \pm 1.88$ and $2.76 \pm 2.31$ ( $P$ value 0.986 ) The mean reticulocyte count was $4.2 \pm 3.4$ and $5.8 \pm 3.8$ ( $P$ value $=0.034$ ), which only showed a difference in reticulocyte count. The convenience was between the two groups. Ritoximab was $9(11.0 \%)$ and $3(11.1 \%)$, respectively, with no significant difference $(p$ value $=0.945)$. According to Cox Proportion Hazard Model, two factors of high age $(P$ value $=0.001)$ and appearance of neurological manifestations $(P$ value of 0.001$)$ were two predictors of long-term mortality in patients. 
Determination of Predictors of Recurrence:

In the two groups with and without recurrence, the frequency of female was 20 (83.3\%) and 60 (66.7\%), respectively $(P$ value $=0.113)$. The mean age of the two groups was 32.4699 .54 years and 41.1315 15.68 years, respectively, which was significantly lower in the relapsed group $(P$ value $=0.011)$. According to the distribution of the disease in two groups with and without recurrence, the frequency of idiopathic type was 10 cases $(41.7 \%)$ and 37 cases $(41.1 \%)$ respectively, the frequency of pregnancy type was 2 cases $(8.3 \%)$ and 19 cases (21.1\%), frequency of immunologic type was 12 cases (50\%) and 19 cases $(21.1 \%)$, transplant type frequency equal to 0 cases $(0.2 \%)$ and 7 cases $(7.8 \%)$. \%), Malignant type was 0 $(0.2 \%)$ and $6(6.7 \%)$, and drug-induced frequency was $0(0.2 \%)$ and $2(2.2 \%)$, respectively. There was a significant difference between the two groups in the frequency of relapsed immunologic type ( $P$ value $=$ 0.042). In terms of clinical manifestations, the frequency of neurologic manifestations was $6(25 \%)$ and $18(20 \%)$ respectively, in the two groups with and without recurrence, hematologic involvement was 16 (66.7\%) and 48 cases (53.3\%), jaundice occurrence $0(0.2 \%)$ and 2 cases (2.2\%), fever occurrence 1 case $(4.2 \%)$ and 0 cases $(0.2 \%)$ Laboratory presentation was equal to $1(4.2 \%)$ and $21(23.3 \%)$ and creatinine increased to $0(0.2 \%)$ and $1(1.1 \%)$, respectively, indicating no difference between the two groups. Was ( $P$ value 0.10$)$. In the group of patients with and without recurrence, mean hemoglobin was $8.26+2.25$ and $9.94+9.64$ ( $P$ value 0.402$)$, mean creatinine was $1.76+1.91$ and $1.45 \%$, respectively. 1.78 ( $P$ value $=$ 0.966), mean LDH was $1929.04+1929.04$ and $2525.45+1361.20$ ( $P$ value $=261.26)$, mean platelet count was 2106.61818.45 And $40611 \pm 11 / 25993$ 82/82 (P value 0.001), AST mean $63.62+65.53$ and $59.55+53.88$ ( $P$ value 0.754 ), ALT mean $77.3+49.0+49.6 \pm 99.0$ ( $P$ value 0.609$)$, mean total bilirubin 2.7 +2.5 and $2.7+2.2$ (P value 0.949$)$ and mean The reticulocyte count was $7.9+4.9$ and $6.7+3.1(P$ value $=$ $0.123)$, which only showed a difference in platelet count between the two groups. It was Roe. Ritoximab was 1 (4.3\%) and 11 (12.8\%), respectively, with no significant difference ( $p$ value 0.148$)$. According to Cox Proportion Hazard Model, three factors of low age ( $P$ value 0.042$)$, immunological category ( $P$ value 0.003 ) and decrease in platelet count ( $p$ value 0.001 ) are predictors of long-term recurrence. The duration was in patients. Table 1 lists the predictors of mortality TTP patient in our study.

Predictors of TTP Death

\begin{tabular}{lllll} 
Factor & Beta multiplier & standard deviation & Probability ratio & P value \\
\hline Older age & 0.056 & 0.020 & 1.058 & 0.006 \\
\hline Malignant Cause & $\mathbf{5 1 6 / 2 0 -}$ & $\mathbf{6 1 1 / 1}$ & 0.001 & 0.999 \\
\hline Neurologic manifestation & $\mathbf{5 8 8 / 1 -}$ & 0.603 & 0.204 & 0.008 \\
\hline The reticulocyte count & $\mathbf{1 0 2 / 0 -}$ & 0.092 & $\mathbf{9 0 3 / 0}$ & 0.263
\end{tabular}

Table 1: Predictors of TTP Death in our study 


\section{Conclusions:}

As a final conclusion, in our study, first, the epidemiological and clinical distribution of TTP patients was entirely in line with studies published by other communities. The first-line treatment of patients with TTP including PEX and rituximab had complete remission of $63.2 \%$, partial remission of $12.3 \%$ and total remission of $75.5 \%$ in our population. However, recurrence was about $21.1 \%$ and long-term mortality equivalent to $26.3 \%$. Long-term mortality in these patients was predictable with factors such as old age and the appearance of neurologic manifestations, whereas younger patients, patients with immunologic disease, and patients with low platelet counts were at greater risk for long-term recurrence. They were facing a long time.

\section{References}

1. Moschcowitz E. Hyaline thrombosis of the terminal arterioles and capillaries: a hitherto undescribed disease. Pros N Patrol Soc. 1924; 24:21-24.

2. Levy GG, Nichols WC, Lain EC, et al. Mutations in a member of the ADAMTS gene family cause thrombotic thrombocytopenic purpura. Nature. 2001; 413(6855):488-494.

3. Scully M, Yarranton $\mathrm{H}$, Leister $\mathrm{R}$, et al. Regional UK TTP registry: correlation with laboratory ADAMTS 13 analysis and clinical features. Br J Haematol. 2008; 142(5):819-826.

4. Lotto LA, Marian M, Canzoni D, et al. Different clinical severity of first episodes and recurrences of thrombotic thrombocytopenic purpura. Br J Haematol. 2010; 151(5):488-494.

5. Fujimura Y, Matsumoto M. Registry of 919 patients with thrombotic microangiopathies across Japan: database of Nara Medical University during 1998-2008. Intern Med. 2010; 49(1):7-15.

6. Kremer Hovinga JA, Vesey SK, Terrell DR, L "amole B, George JN. Survival and relapse in patients with thrombotic thrombocytopenic purpura. Blood. 2010; 115(8):1500-1511.

7. Jang MJ, Chong SY, Kim I-H, et al. Clinical features of severe acquired ADAMTS13 deficiency in thrombotic thrombocytopenic purpura: the Korean TTP registry experience. IN J Hematol. 2011; 93(2):163-169.

8. DE ford CC, Reese JA, Schwartz LH, et al. multiple major morbidities and increased mortality during long-term follow-up after recovery from thrombotic thrombocytopenic purpura. Blood. 2013; 122(12):2023-2029, quiz 2142.

9. Bomber P, Kivivali L, Pepperell D, et al; TTP registry steering committee. Diagnosis and management of thrombotic thrombocytopenic purpura (TTP) in Australia: findings from the first 5 years of the Australian TTP/thrombotic microangiopathy registry. Intern Med J. 2016; 46(1):71-79. 10. Mariotte E, Azoulay E, Galicier L, et al; French Reference Center for Thrombotic Microangiopathies. Epidemiology 
and pathophysiology of adulthood-onset thrombotic microangiopathy with severe ADAMTS13 deficiency (thrombotic thrombocytopenic purpura): a cross-sectional analysis of the French national registry for thrombotic microangiopathy. Lancet Haematol. 2016; 3(5): e237-e245.

10. George JN, Al-Nouri ZL. Diagnostic and therapeutic challenges in the thrombotic thrombocytopenic purpura and hemolytic uremic syndromes. Hematology Am Sock Hematol Educe Program. 2012; 2012:604-609.

11. Reese JA, Maharajah DS, Kremer Hovinga JA, Vesey SK, Terrell DR, George JN. Children and adults with thrombotic thrombocytopenic purpura associated with severe, acquired Adamts 13 deficiency: comparison of incidence, demographic and clinical features. Pediatric Blood Cancer. 2013; 60(10):1676-1682.

12. Jolly BS, Cope P, Veyradier A. Child- and adolescent-onset acquired thrombotic thrombocytopenic purpura with severely deficient ADAMTS13: a cohort study of 45 cases from the French Thrombotic Microangiopathies Registry. Lancet Haematol. 2016; 3(11): e537-e546.

13. Sadler JE. What's new in the diagnosis and pathophysiology of thrombotic thrombocytopenic purpura Hematology Am Sock Hematol educe Program 2015; 2015:631-636

14. Moake JL, Rudy CK, and Troll JH, et al. Unusually large plasma factor VIII: von Will brand factor multimers in chronic relapsing thrombotic thrombocytopenic purpura. N Engle J Med. 1982; 307(23):1432-1435.

15. Asada Y, Sumiyoshi A, Hayashi T, Suzumiya J, and Kaketani K. Immunohistochemistry of vascular lesion in thrombotic thrombocytopenic purpura, with special reference to factor VIII related antigen. Thromb Res. 1985; 38(5):469-479.

16. Rock GA, Sumac KH, Buskard NA, et al; Canadian Apheresis Study Group. Comparison of plasma exchange with plasma infusion in the treatment of thrombotic thrombocytopenic purpura. $\mathrm{N}$ Eng. $J$ Med. 1991; 325(6):393-397.

17. Furan $M$, Robles $R$, $L$ "ammle B. Partial purification and characterization of a protease from human plasma cleaving von Will brand factor to fragments produced by in vivo proteolysis. Blood. 1996; 87(10):4223-4234.

18. Furan $M$, Robles $R$, Galbusera $M$, et al. von Will brand factor-cleaving protease in thrombotic thrombocytopenic purpura and the hemolytic-uremic syndrome. N Eng. J Med. 1998; 339(22):15781584.

19. Tsai HM, Lain EC. Antibodies to von Will brand factor-cleaving protease in acute thrombotic thrombocytopenic purpura. N Eng. J Med. 1998; 339(22):1585-1594.

20. Sajama K, Mimura N, Hiroshima M, et al. A novel human metalloprotease synthesized in the liver and secreted into the blood: possibly, the von Will brand factor-cleaving protease? J Biochem. 2001; 130(4):475-480.

21. Veyradier A, Obert B, Houllier A, Meyer D, Girma JP. Specific von Will brand factor-cleaving protease in thrombotic microangiopathies: a study of 111 cases. Blood. 2001; 98(6): 1765-1772. 
22. Bianchi V, Robles R, Alberio L, and Furan M, L "ammle B. Von Will brand factor-cleaving protease (ADAMTS13) in thrombocytopenic disorders: a severely deficient activity is specific for thrombotic thrombocytopenic purpura. Blood. 2002; 100(2):710-713.

23. Zheng XL, Kaufman RM, Goodnough LT, Sadler JE. Effect of plasma exchange on plasma ADAMTS13 metalloprotease activity, inhibitor level, and clinical outcome in patients with idiopathic and no idiopathic thrombotic thrombocytopenic purpura. Blood. 2004; 103(11): 4043-4049.

24. Verbij FC, Fijnheer R, Voorberg J, and Sorvillo N. Acquired TTP: ADAMTS13 meets the immune system. Blood Rev. 2014; 28(6):227-234.

25. Klaus C, Plaimauer B, Studt J-D, et al. Epitope mapping of ADAMTS13 autoantibodies in acquired thrombotic thrombocytopenic purpura. Blood. 2004; 103(12):4514-4519.

26. Ferrari S, Scheiflinger F, Rieger M, et al; French Clinical and Biological Network on Adult Thrombotic Microangiopathies. Prognostic value of anti-ADAMTS 13 antibody features (Ig isotype, titer, and inhibitory effect) in a cohort of 35 adult French patients undergoing a first episode of thrombotic microangiopathy with undetectable ADAMTS 13 activity. Blood. 2007; 109(7):2815-2822. 28. Lotta LA, Valsecchi C, Pontiggia S, et al. Measurement and prevalence of circulating ADAMTS13-specific immune complexes in autoimmune thrombotic thrombocytopenic purpura. J Thromb Haemost. 2014; 12(3): 329-336.

27. Uemura M, Fujimura $Y$, Matsumoto $M$, et al. Comprehensive analysis of ADAMTS13 in patients with liver cirrhosis. Thromb Haemost. 2008; 99(6):1019-1029.

28. Studt J-D, Kremer Hovinga JA, Antoine G, et al. Fatal congenital thrombotic thrombocytopenic purpura with apparent ADAMTS13 inhibitor: in vitro inhibition of ADAMTS13 activity by hemoglobin. Blood. 2005; 105(2):542-544.

29. Ono T, Mimuro J, Madoiwa S, et al. Severe secondary deficiency of von Will brand factor cleaving protease (ADAMTS13) in patients with sepsis-induced disseminated intravascular coagulation: its correlation with development of renal failure. Blood. 2006; 107(2):528-534.

30. Paige V, Azoulay E, Coquet I, et al. The prognostic value of ADAMTS13 (a disinterring and metalloprotease with thrombospondin type 1 repeats, member 13) deficiency in septic shock patients involves interleukin- 6 and is not dependent on disseminated intravascular coagulation. Crist Care. 2013; 17(6):R273.

31. Lotta LA, Garagiola I, Pella R, Cairo A, Peyvandi F. ADAMTS13 mutations and polymorphisms in congenital thrombotic thrombocytopenic purpura. Hum Mutat. 2010;31(1):11-19.

32. Hanby HA, Zheng XL. Current status in diagnosis and treatment of hereditary thrombotic thrombocytopenic purpura. Hereditary Genet. 2014; 3(1):e108.

33. Camilleri RS, Cohen H, Mackie IJ, et al. Prevalence of the ADAMTS-13 missense mutation R1060W in late onset adult thrombotic thrombocytopenic purpura. J Thromb Haemost. 2008; 6(2):331-338. 36. Matte-Cohen M, Garret C, Wolf M, et al; French Reference Center for Thrombotic Microangiopathies. Unexpected frequency of Upshaw-Schulman syndrome in pregnancy onset thrombotic thrombocytopenic purpura. Blood. 2012; 119(24):5888-5897. 
34. Motto DG, Chauhan AK, Zhu G, et al. Shigatoxin triggers thrombotic thrombocytopenic purpura in genetically susceptible ADAMTS13-deficient mice. J Clan Invest. 2005; 115(10):2752-2761.

35. Vanhoorelbeke K, De Meyer SF. Animal models for thrombotic thrombocytopenic purpura. J Thromb Haemost. 2013; 11(Supple 1):2-10.

36. Page EE, Kremer Hovinga JA, Terrell DR, Vesey SK, George JN. Clinical importance of ADAMTS13 activity during remission in patients with acquired thrombotic thrombocytopenic purpura. Blood. 2016; 128(17):2175-2178.

37. Terrell DR, Motto DG, Kremer Hovinga JA, L “ammle B, George JN, Vesely SK. Blood group $O$ and black race are independent risk factors for thrombotic thrombocytopenic purpura associated with severe ADAMTS13 deficiency. Transfusion. 2011; 51(10):2237-2243.

38. Martino S, Jamme M, Deligny C, et al; French Reference Center for Thrombotic Microangiopathies. Thrombotic thrombocytopenic purpura in black people: impact of ethnicity on survival and genetic risk factors. PLoS One. 2016; 11(7):e0156679.

39. Coppo P, Busson M, Veyradier A, et al; French Reference Centre for Thrombotic Microangiopathies. HLA-DRB1*11: a strong risk factor for acquired severe ADAMTS13 deficiency-related idiopathic thrombotic thrombocytopenic purpura in Caucasians. J Thromb Haemost. 2010; 8(4):856-859.

40. Mannucci PM, Canciani MT, Forza I, Lussana F, Latitude A, Rossi E. Changes in health and disease of the metalloprotease that cleaves von Will brand factor. Blood. 2001; 98(9):2730-2735.

41. George JN, Vesely SK, Terrell DR. The Oklahoma Thrombotic Thrombocytopenic Purpura-Hemolytic Uremic Syndrome (TTPHUS) Registry: a community perspective of patients with clinically diagnosed TTP-HUS. Semin Hematol. 2004; 41(1):60-67.

42. Benhamou Y, Belle P-Y, Baden B, et al; Reference Center for Thrombotic Microangiopathies; Experience of the French Thrombotic Microangiopathies Reference Center. Cardiac troponin-I on diagnosis predicts early death and refractoriness in acquired thrombotic thrombocytopenic purpura. J Thromb Haemost. 2015; 13(2):293-302.

43. Zafrani $L$, Mariotte $E$, Darmon $M$, et al. Acute renal failure is prevalent in patients with thrombotic thrombocytopenic purpura associated with low plasma ADAMTS13 activity. J Thromb Haemost. 2015; 13(3):380-389.

44. Noris M, Remuzzi G. Atypical hemolytic-uremic syndrome. N Engl J Med. 2009; 361(17): 1676-1687.

45. Stepanian A, Cohen-Moatti M, Sanglier T, et al; ECLAXIR Study Group. Von Will brand factor and ADAMTS13: a candidate couple for preeclampsia pathophysiology. Arterioscler Thromb Vasc Biol. 2011;31(7):1703-1709.

46. Veyradier A, Meyer D. Thrombotic thrombocytopenic purpura and its diagnosis. J Thromb Haemost. 2005; 3(11):2420-2427.

47. Coppo P, Schwarzenegger M, Buffet M, et al; French Reference Center for Thrombotic Microangiopathies. Predictive features of severe acquired ADAMTS13 deficiency in idiopathic thrombotic microangiopathies: the French TMA reference center experience. PLoS One. 2010; 5(4):e10208. 
48. Bentley MJ, Wilson AR, Rodgers GM. Performance of a clinical prediction score for thrombotic thrombocytopenic purpura in an independent cohort. Vox Sang. 2013; 105(4): 313-318.

49. Obert B, Tout H, Veyradier A, Fressinaud E, Meyer D, Girma JP. Estimation of the von Will brand factorcleaving protease in plasma using monoclonal antibodies to vWF. Thromb Haemost. 1999; 82(5):1382-1385.

50. Garretson HE, Turecek PL, Schwarz HP, L “ammle B, Furan M. Assay of von Will brand factor (vWF)cleaving protease based on decreased collagen binding affinity of degraded vWF: a tool for the diagnosis of thrombotic thrombocytopenic purpura (TTP). Thromb Haemost. 1999; 82(5): 13861389.

51. Kokame K, Nobe Y, Kokako Y, Okayama A, Miyata T. FRETS-VWF73, a first fluorogenic substrate for ADAMTS13 assay. Br J Haematol. 2005; 129(1):93-100.

52. Thouzeau S, Capdenat S, St 'epanian A, Coppo P, Veyradier A. Evaluation of a commercial assay for ADAMTS13 activity measurement. Thromb Haemost. 2013; 110(4):852-853.

53. Jolly B, Stepanian A, Hajage D, et al. Evaluation of a chromogenic commercial assay using VWF-73 peptide for ADAMTS13 activity measurement. Thromb Res. 2014; 134(5): 1074-1080.

54. Hubbard AR, Heath AB, Kremer Hovinga JA; Subcommittee on von Willebrand Factor. Establishment of the WHO 1st International Standard ADAMTS13, plasma (12/252): communication from the SSC of the ISTH. J Thromb Haemost. 2015; 13(6):1151-1153.

55. Meyer SC, Sulzer I, L “ammle B, Kremer Hovinga JA. Hyperbilirubinemia interferes with ADAMTS13 activity measurement by FRETS-VWF73 assay: diagnostic relevance in patients suffering from acute thrombotic microangiopathies. J Thromb Haemost. 2007; 5(4):866-867.

56. Knavish MA, Farmland A, Owen J. Long-term management of acquired thrombotic thrombocytopenic purpura using serial plasma ADAMTS13 measurements. Eur J Haematol. 2012; 88(6):518-525.

57. Mancini I, Valsecchi C, Lotta LA, et al. FRETS-VWF73 rather than CBA assay reflects ADAMTS13 photolytic activity in acquired thrombotic thrombocytopenic purpura patients. Thromb Haemost. 2014; $112(2): 297-303$.

58. Connell NT, Chives T, Sweeney JD. Effect of ADAMTS13 activity turnaround time on plasma utilization for suspected thrombotic thrombocytopenic purpura. Transfusion. 2016; 56(2):354-359. 62. Mackie I, Langley K, Chitolie A, et al. Discrepancies between ADAMTS13 activities assays in patients with thrombotic microangiopathies. Thromb Haemost. 2013; 109(3):488-496.

59. Coppo P, Froissart A, French Reference Center for Thrombotic Microangiopathies. Treatment of thrombotic thrombocytopenic purpura beyond therapeutic plasma exchange. Hematology Am Soc Hematol educe Program. 2015; 2015: 637-643.

60. Vesely SK, George JN, L “ammle B, et al. ADAMTS13 activity in thrombotic thrombocytopenic purpura-hemolytic uremic syndrome: relation to presenting features and clinical outcomes in a prospective cohort of 142 patients. Blood. 2003; 102(1):60-68.

61. Hughes C, McEwan JR, Longair I, et al. Cardiac involvement in acute thrombotic thrombocytopenic purpura: association with troponin T and IgG antibodies to ADAMTS 13. J Thromb Haemost. 2009; 
$7(4): 529-536$.

62. Benhamou Y, Assi 'e C, Boelle P-Y, et al; Thrombotic Microangiopathies Reference Center. Development and validation of a predictive model for death in acquired severe ADAMTS13 deficiency-associated idiopathic thrombotic thrombocytopenic purpura: the French TMA Reference Center experience. Haematologica. 2012; 97(8):1181-1186.

63. George JN. How I treat patients with thrombotic thrombocytopenic purpura: 2010. Blood. 2010; 116(20):4060-4069.

64. Lewis QF, Lanneau MS, Mathias SD, Terrell DR, Vesely SK, George JN. Long-term deficits in healthrelated quality of life after recovery from thrombotic thrombocytopenic purpura. Transfusion. 2009; 49(1):118-124.

65. Hie M, Gay J, Galicier L, et al; French Thrombotic Microangiopathies Reference Centre. Preemptive rituximab infusions after remission efficiently prevent relapses in acquired thrombotic thrombocytopenic purpura. Blood. 2014; 124(2):204-210.

66. Sarode R, Bandarenko N, Brecher ME, et al. Thrombotic thrombocytopenic purpura: 2012 American Society for Apheresis (ASFA) consensus conference on classification, diagnosis, management, and future research. J Clin Apher. 2014; 29(3):148-167.

67. Scully M, Hunt BJ, Benjamin S, et al; British Committee for Standards in Haematology. Guidelines on the diagnosis and management of thrombotic thrombocytopenic purpura and other thrombotic microangiopathies. Br J Haematol. 2012; 158(3):323-335.

68. Froissart A, Buffet M, Veyradier A, et al; French Thrombotic Microangiopathies Reference Center; Experience of the French Thrombotic Microangiopathies Reference Center. Efficacy and safety of first-line rituximab in severe, acquired thrombotic thrombocytopenic purpura with a suboptimal response to plasma exchange. Crit Care Med. 2012; 40(1):104-111.

69. Toussaint-Hacquard M, Coppo P, Soudant M, et al. Type of plasma preparation used for plasma exchange and clinical outcome of adult patients with acquired idiopathic thrombotic thrombocytopenic purpura: a French retrospective multicenter cohort study. Transfusion. 2015; 55(10):2445-2451.

70. Rock G, Shumak KH, Sutton DM, Buskard NA, Nair RC; Members of the Canadian Apheresis Group. Cry supernatant as replacement fluid for plasma exchange in thrombotic thrombocytopenic purpura. Br J Haematol. 1996; 94(2):383-386.

71. Zeigler ZR, Shadduck RK, Gryn JF, et al; North American TTP Group. Cryoprecipitate poor plasma does not improve early response in primary adult thrombotic thrombocytopenic purpura (TTP). J Clin Apher. 2001; 16(1):19-22.

72. Balduini CL, Gugliotta L, Luppi M, et al; Italian TTP Study Group. High versus standard dose methylprednisolone in the acute phase of idiopathic thrombotic thrombocytopenic purpura: a randomized study. Ann Hematol. 2010; 89(6): 591-596.

73. Bell WR, Braine HG, Ness PM, Kickler TS. Improved survival in thrombotic thrombocytopenic purpurahemolytic uremic syndrome. Clinical experience in 108 patients. N Engl J Med. 1991; 325(6):398-403. 
74. Elliott MA, Heit JA, Pruthi RK, Gastineau DA, Winters JL, Hook CC. Rituximab for refractory and or relapsing thrombotic thrombocytopenic purpura related to immune-mediated severe ADAMTS13deficiency: a report of four cases and a systematic review of the literature. Eur J Haematol. 2009; 83(4):365-372.

75. Scully M, McDonald V, Cavenagh J, et al. A phase 2 study of the safety and efficacy of rituximab with plasma exchange in acute acquired thrombotic thrombocytopenic purpura. Blood. 2011; 118(7):1746-1753.

76. Jasti S, Coyle T, Gentile T, Rosales L, Poiesz B. Rituximab as an adjunct to plasma exchange in TTP: a report of 12 cases and review of literature. J Clin Apher. 2008; 23(5):151-156.

77. Ling HT, Field JJ, Blinder MA. Sustained response with rituximab in patients with thrombotic thrombocytopenic purpura: a report of 13 cases and review of the literature. Am J Hematol. 2009; 84(7):418-421.

78. de la Rubia J, Moscard 'o F, G 'omez MJ, et al; Grupo Esp. no de Af 'eresis. Efficacy and safety of rituximab in adult patients with idiopathic relapsing or refractory thrombotic thrombocytopenic purpura: results of a Spanish multicenter study. Transfus Apheresis Sci. 2010; 43(3):299-303.

79. Page EE, Kremer Hovinga JA, Terrell DR, Vesely SK, George JN. Rituximab reduces risk for relapse in patients with thrombotic thrombocytopenic purpura. Blood. 2016; 127(24):3092-3094.

80. Scully M, Cohen H, Cavenagh J, et al. Remission in acute refractory and relapsing thrombotic thrombocytopenic purpura following rituximab is associated with a reduction in IgG antibodies to ADAMTS-13. Br J Haematol. 2007; 136(3):451-461.

81. Catalan SR, Wu HM. Acquired thrombotic thrombocytopenic purpura: new therapeutic options and their optimal use. J Thromb Haemost. 2015; 13(supple 1):S223-S229.

82. Chen J, Rahman A, Gush ken FC, et al. Nacetylcysteine reduces the size and activity of von Willebrand factor in human plasma and mice. J Clin Invest. 2011; 121(2):593-603.

83. Short J, Oh DH, and Opal SS. ADAMTS13 antibody depletion by bortezomib in thrombotic thrombocytopenic purpura. N Engl J Med. 2013; 368(1):90-92.

84. Plaimauer B, Kremer Hovinga JA, Juno C, et al. Recombinant ADAMTS13 normalizes von Willebrand factor-cleaving activity in plasma of acquired TTP patients by overriding inhibitory antibodies. J Thromb Haemost. 2011; 9(5): 936-944.

85. Schiviz A, Wuersch K, Piskernik C, et al. A new mouse model mimicking thrombotic thrombocytopenic purpura: correction of symptoms by recombinant human ADAMTS13. Blood. 2012; 119(25):6128-6135.

86. Peyvandi F, Scully M, Kremer Hovinga JA, et al; TITAN Investigators. Caplacizumab for Acquired Thrombotic Thrombocytopenic Purpura. N Engl J Med. 2016; 374(6):511-522.

87. Swart L1, Schapkaitz E1, Mahlangu JN1. Thrombotic thrombocytopenic purpura: A 5-year tertiary care center experience. J Clin Apher. 2019 Feb; 34(1):44-50. Doi: 10.1002/jca.21673. Epub 2018 Dec 8. 
88. Staley EM1, Cao W1, Pham HP2, Kim CH3, Kocher NK1, Zheng L1, Gangaraju R4, Lorenz RG1, Williams LA1, Marques MB1, Zheng XL5. Clinical factors and biomarkers predict outcome in patients with immune-mediated thrombotic thrombocytopenic purpura. Haematologica. 2019 Jan; 104(1):166-175. Doi: 10.3324/haematol.2018.198275. Epub 2018 Aug 31.

89. Alwen F1, Vendramin C2, Vanhoorelbeke K3, Langley K2, McDonald V4, Austin S5, Clark A6, Lester W7, Gooding R8, Biss T9, Dutta T10, Cooper N11, Chapman 012, Cranfield T13, Douglas K14, Watson HG15, van Vern JJ16, Sibson K17, Thomas W18, Manson L19, Hill QA20, Benjamin S21, Ellis D1, Westwood JP1, Thomas M1,22, Scully M1,22. Presenting ADAMTS13 antibody and antigen levels predict prognosis in immune-mediated thrombotic thrombocytopenic purpura. Blood. $2017 \mathrm{Jul}$ 27; 130(4):466-471. Doi: 10.1182/blood-2016-12-758656. Epub 2017 Jun 2.

90. Iqbal S1, Zaidi SZ2, Motabi IH3, Alshehry NF4, AlGhamdi MS5, Tailor IK6. Thrombotic thrombocytopenic purpura - analysis of clinical features, laboratory characteristics and therapeutic outcome of 24 patients treated at a Tertiary Care Center in Saudi Arabia. Pak J Med Sci. 2016 NovDec; 32(6):1494-1499. Doi: 10.12669/pjms.326.11274.

91. Wang Y1, Zhang JY2, Nil ZY1, Lin FR1, Zhou JH3. Clinical analysis of 14 patients with thrombotic thrombocytopenic purpura. Zhongguo Shi Yan Xu Ye Xu Zac Zhi. 2014 Apr; 22(2):407-11. Doi: 10.7534/j.issn.1009-2137.2014.02.026.

92. Sun L1, Yu Z1, Bu Y1, Su J1, Wang C1, Cao L1, Bai X1, Wang Z1, Ruan C1. The clinical studies of 51 patients with thrombotic thrombocytopenic purpura. Songhua Xu Ye Xue Zac Zhi. 2014 Feb; 35(2):147-51. Doi: 10.3760/cma.j.issn.0253-2727.2014.02.019.

93. El-Husseiny NM1, Goubran H, Fahmy HM, Tawfik NM, Moustafa H, Amin SN, El-Ekiaby M. Outcome and relapse risks of thrombotic thrombocytopaenic purpura: an Egyptian experience. Postgrad Med J. 2012 Aug; 88(1042):433-6. Do: 10.1136/postgradmedj-2011-130375. Epub 2012 Mar 20.

94. Chaturvedi, Shruti, et al. "Management and outcomes for patients with TTP: analysis of 100 cases at a single institution." American journal of hematology 88.7 (2013): 560-565.

95. Frawley, Natasha, et al. "Thrombotic thrombocytopenic purpura is associated with a high relapse rate after plasma exchange: a single-centre experience." Internal medicine journal 39.1 (2009): 19-24

\section{Declarations}

Author Contribution section:

1) Mohammad - Reza Rostami MD

Hematology and Medical oncology Fellow, Tehran University of Medical sciences, Tehran, Iran 35percent Contribution share 
2)Amirmasoud Kazemzadeh Houjaghan , Intern of Tehran University of Medical sciences, Tehran, Iran 28percent Contribution share

3)Sahar Tavakoli Shiraji MD Assistant Professor of Hematology, oncology and Bone Marrow Transplantation, Tehran University of Medical sciences, Tehran, Iran 22percent Contribution share 4) Hosein Kamranzadeh Fumani MD Assistant Professor of Hematology , oncology and Stem cell Transplantation Research center , Tehran University of Medical sciences, Tehran, Iran 15percent Contribution share

We, the authors of this article, Dr. Mohammad Reza Rostami, Dr. Amir Massoud Kazemzadeh Hojjahan, Dr. Sahar Tavakoli Shiraji and Dr. Hossein Kamranzadeh Foumani, expressly declare that we have had no conflict of interest in carrying out this project and article, respectively.

Four bullet points:

Background: thrombotic thrombocytopenic purpura (TTP) is associated with microangiopathic hemolytic anemia, thrombocytopenia, and micro vascular thrombus plus fever, fluctuating neurologic abnormalities and renal damage.

Method: The study was a retrospective cohort study in patient with TTP in Shariati hospital from 2010 to 2017. 114 subjects with TTP after rule out of other microangiopathic hemolytic anemia were included in this study.

Results: 114 case of TTP ( 80 female and 34 male) were identified. Mean ages of participated in the study were 39 years. Hematologic and neurologic symptoms were most common manifestations. Laboratory result at the time of diagnosis revealed mean thrombocytopenia (PIt: 29100), anemia ( $\mathrm{Hb}: 8 / 1)$, elevated LDH (15100).

Conclusion: in difference clinical symptom and laboratory finding may be predict mortality and occurrence of relapse 